\title{
Spoken dialogue BIM systems - an application of Big Data in construction
}

\begin{abstract}
Purpose: With the rapid development in the internet technologies, the applications of big data in construction have seen considerable attention. Currently, there are many input/output modes of capturing construction knowledge related to all construction stages. On the other hand, BIM systems have been developed to help in storing various structured data of buildings. However, these systems cannot fully capture the knowledge and unstructured data utilised in the operation of building systems in a usable format that utilises the intelligent capabilities of BIM systems. Therefore, this research aims to adopt the concept of Big Data and develop a Spoken Dialogue BIM system to capture buildings operation knowledge; particularly for building maintenance and refurbishment.

Design/methodology/approach: The proposed system integrates cloud-based spoken dialogue system and case-based reasoning BIM system.

Findings: The system acts as an interactive expert agent that seeks answers from the user for questions specific to building maintenance problems and help searching for solutions from previously stored knowledge cases. The practices of monitoring and maintaining buildings performance can be more efficient by the retrieval of relevant solutions from the captured knowledge to new problems when maintaining buildings components. The developed system enables easier capture and search for solutions to new problems with a more comprehensive retrieval of information.

Originality/value: Capturing multi-modes data into BIM systems using the cloud-based spoken dialogue systems will help construction teams utilise the high volume of data generated over building lifecycle and search for the most suitable solutions for maintenance problems. This new area of research also contributes to the current BIM systems by advancing their capabilities to instantly capture and retrieve knowledge of operations instead of only information.
\end{abstract}

Keywords: Big data, BIM, Spoken Dialogue Systems, Knowledge systems

\section{Introduction}

Different types of information are generated over the various phases of building's lifecycle including the maintenance of the various complex systems used by buildings' occupants. As the generation and collection of building information is commonly fragmented, created over the building's lifecycle by different teams with different objectives, stored in different systems, the integration of this information is a complex process. This process has indicated to the relevance of big data to the construction industry, particularly in relation to the volume and variety of data where structured and unstructured data is being generated for each construction project. While many industrial sectors such as the health and transport sectors have started to use big data resources, the construction sector is still behind. McKinsey Global Institute's (MGI) studied big data in: healthcare in the United States, the public sector in Europe, retail in the United States, and manufacturing and personal-location data globally. On the MGI's big data value potential index, the construction sector is between low and high potential value. Using data in the construction industry for the period 2000-2008, MGI (2011) reported that construction has the least stored data if compared with the Manufacturing and Government sectors. In this respect, the UK government has launched few initiatives, such as the Smart Cities Forum, the Open Data Institute and the Future Cities Catapult to support this field in AEC sector. Shrestha (2013) explored the possible applications of big data in the 
construction industry and indicated to how the big data predictive analytics can be used for asset management, maintenance, schedule analysis, performance evaluation of the subcontractors, and in improving safety of the construction work zone. The use of big data needs the industry to prepare the necessary infrastructure for storing and analysing those data which in turn, will help construction teams make more informed data-driven decisions.

The importance of Building Management (BM) is shown in the rapid development of the business environment in both private and public sectors, especially with the growing demand to reduce energy consumption and $\mathrm{CO}_{2}$ emissions. $\mathrm{BM}$ includes various decisions, policies and processes which are largely influenced by non-financial aspects and expectations of various and authoritative stakeholders to improve the delivery of services, Barrett and Baldry (2003). Within the processes of BM, building maintenance is a major part which can be either preventive (routine maintenance plan) or corrective (reactive maintenance to elements break down). There is always a need to have sufficient information on products ready available for any maintenance operation in addition to related knowledge and past experience of maintenance cases to help conduct new work. The principles behind capturing such information and knowledge about the various project life cycle phases, including BM, have moved from manual recording to the use of IT solutions such as Radio Frequency Identification (RFID) (as the work of Legner and Thiesse (2006) and Chien-Ho Ko (2009)) to collect and share maintenance data with the most minimal manual data entry. These efforts, in addition to others, drove to the development of a more generic IT solutions especially with the development of Building Information Modelling (BIM) and more recently with the use of Internet of Things and Big Data.

On the other hand, despite the beneficial insights of applying big data analytics, they are still relatively immature in terms of functionality and robustness, McMalcolm (2015). Therefore, more advanced big data analytics tools are required which are to be more relevant to construction industry. In addition, with the recent development in BIM, the research in this area tends to establish the relationship between big data and BIM.

BIM as a process and technology can provide greater consistency of structured data for construction. However, the current BIM systems should be developed further to incorporate non-technical and non-geometric knowledge associated with building practices in addition to technical and geometric data. In this respect, Knowledge Systems, as special tools among the big data analytics, usually capture the experiences of professionals and store pervious operational cases with all lessons learned and solutions adopted to be used later as guidance on solutions for new problems.

However, capturing knowledge in digital format is always the problem of developing a sustained knowledge system that expands over time by adding more knowledge cases over the whole lifecycle of a building. With the current development in data capturing tools and devices such as mobile technology and internet facilities, knowledge can be captured in a more effective way.

From the above summary, this research aims to establish the position of BIM models within the Big Data principles and use a data capture technique (Spoken Dialogue Systems (SDS)) to integrate the knowledge of building operations into BIM systems. The research methodology adopted to achieve this aim included the following stages:

1. Reviewing the use of Big Data and SDS in construction 
2. Investigating the structured and unstructured data of the building maintenance and operation stage to develop a basic ontology for building maintenance

3. Developing a knowledge-based analytics tool to analyse the captured knowledge for future use by buildings teams

4. Integrating the analytics tool with BIM environment

5. Developing a SDS module to be integrated with the developed system

6. Validating the application of the developed system using a case study.

While literature review was the main task to conduct stage 1, the data needed for stage 2 was collected from various maintenance related documents and also by meeting with buildings professionals to outline the proposed ontology. Further details on this data and the other stages will be illustrated later in the paper. The following sections in this paper will introduce Big Data for construction, review the potentials of using SDS in capturing building knowledge. The architecture of a proposed Spoken dialogue BIM system as an application of Big Data in construction will be then illustrated with an example of its use.

\section{Big Data for construction}

"Big data" as defined in the Oxford English Dictionary (2015) is the "Extremely large data sets that may be analysed computationally to reveal patterns, trends, and associations, especially relating to human behaviour and interactions". Big Data is always defined in terms of the four Vs: Volume (the amount of data), Velocity (timely data processing and analysing), Variety (data from a range of sources), and Veracity (data integrity).

Mello et al (2014) reported that there are four steps to big data processing: (1) acquisition (data capturing from different sources); (2) access (data indexing, storage, sharing, and archiving); (3) analytics (data analysis and manipulation); and (4) application (making decisions and taking actions). In this respect, big data considers new techniques and tools for data acquisition, access, analytics and application to transform this data into useful information in a timely manner to provide business benefit for the companies (Courtney (2012), Tien (2013), and Waller and Fawcett (2013)). Big data analytics have taken much attention in both the academia and companies of different economic sectors over the past two decades, Chen et al (2012). In general, the big data analytics process the unstructured data which may include various data mining, data optimisation and search techniques. With the use of cloud-computing and knowledge-based solutions, processing this data can enable better use of the variety of data captured.

For the AEC sector, Big Data applications can transform the practices of design, construction, and operation of projects. The available variety of data collection tools such as: mobile devices, social media, smart meters, sensors, satellites, camera footage, traffic flow reports, etc. collect data on performance and behaviour of operations, which can provide huge feedback data from buildings occupants and teams of projects. This can help design and operation teams better understand occupants and users needs, and also help operate buildings, spaces, and services more efficiently. These data capture tools can now provide granular realtime data about utilization patterns which can improve the maintenance of buildings services and operations. The use of social media for example is no longer for non-work communications only. Many business professionals are using social media for sharing business knowledge. The sentiment analysis which is a form of textual analysis of social media can be then used to process these knowledge. 
As big data may include both structured and unstructured data, it is worth noting the difference and classification of these data types, especially in terms of analysis methodologies. It can be noted that majority of the existing analytics tools deal with structured data. Structured data can be generally specified by set of rules within a database, Caldas et al. (2005). However, unstructured data may include various data format like text, images and audio data without specified rules. Parise et al (2012) classified those types of data as: transactional (structured) and non-transactional (unstructured) data. They identified "Crowdsourcing" as the process for collecting unstructured data from a large community or distributed group of people, by which idea generation can be conducted. Crowdsourcing enables companies to pose questions to the community about its services. In terms of analysis, the Textual Analysis, which is computer algorithms that analyse natural language, can help extract topics from text along with their linkages.

Non-transactional data can be also analysed by Decision Science to determine the value, validity, and feasibility of the data and how it is useful for actions. The techniques used in decision science include, for example, listening tools that perform text and sentiment analysis. However, the current listening tools still need further development to improve its accuracy, Parise et al (2012). There is always a need to train these tools on industry-specific keywords for an accurate textual and sentiment analysis.

\section{Big Data and BIM}

As Big Data can be used to improve the development of the next generation of buildings and services, BIM will be a major factor in this development. BIM is largely about structured data and allow the access to multiple databases. However, there is a large volume of unstructured data/information exchanged within construction industry via dialogue conversation or text between team coordinators. Addor and Santos (2014) reported that visualise floor plans and writing down text are the most frequent methods of information exchanges in meeting rooms. Moreover, the amount of unstructured data during construction and the operation stages of buildings increase with the life cycle of the buildings. This non-structured data uses different advanced systems for entry and analysis. The integrated handling of structured and nonstructured data is about the methodology to support two kinds of data systems: model-based systems and document-based systems (Caldas et al., 2005). Some studies have argued that it is possible to link unstructured substances to model-based information systems (Mao et al., 2007, Zhu et al., 2007, Jiao et al., 2013, Li et al., 2014).

As this research aims to establish the position of BIM systems within the big data framework, BIM-based systems have been considered for the analytics of structured data of buildings that need to be integrated with various cloud-based systems and advanced analytics tools for the unstructured data. The following section will first review the available literature about BIM and the analytic knowledge systems applied for building maintenance, as the scope targeted by this research.

\section{Knowledge-based BIM systems}

For the building operation stage, as the main focus of this paper, there are several BIMrelated studies aimed at improving Facilities Management (FM) practices which include buildings maintenance and refurbishment for the functions of locating components, facilitating access of real-time data, checking maintainability, automatic creation of digital assets, quality control and assurance, energy management, and space management (BecerikGerber et al. 2012). Among these applications there are: BIM based package for the FM 
Exemplar project of Sydney Opera House (Akhurst and Gillespie 2006), AROMA-FF which is developed to utilise data including BIM databases to obtain information and geometric representation of facilities and equipment (Lee and Akin 2011), and the web-based Facilities Maintenance Management prototype decision support system (Hao et al. 2010). As all such systems are BIM-based, they mainly utilise structured data. However, they generally do not consider the kind of the unstructured knowledge gained during the operation of buildings. Current BIM related systems mainly focus on capturing technical information and allowing the access to multiple rationale databases. However, capturing and retrieving the knowledge of the maintenance and operation stages needs different technical ontologies from what is typically utilised when developing BIM databases. With further development of BIM systems, researchers such as Motawa and Carter (2013) recommended that knowledge of buildings operations should be also incorporated with the traditional BIM systems to transform the typical information model of buildings (as it is currently developed) into innovative knowledge models.

On the other hand for Knowledge Management (KM), several KM systems have been revolving around managing knowledge in construction and FM. The concept of KM has been utilised to improve performance, reduce cost, increase efficiency and quality. Ali et al. (2002 and 2004) introduced a prototype KM system to improve the management of Reactive Maintenance projects. Other examples include: the web-based system "Consulting Knowledge System" by Lepkova and Bigelis (2007), and "Building Maintenance Community of Practice" by Fong and Wong (2009). The chief objective of such applications is the improvement of knowledge sharing and communication between stakeholders. As new levels of efficiency in sharing information and knowledge have been emerged, the integration of BIM systems with knowledge management tools to capture and retain the operational knowledge has been proposed, Motawa and Almarshad (2013). Therefore, this has established the need to consider various types of data that can be collected from various sources using various tools which the characteristic of big data; "data variety", is mainly about. Therefore, this research proposes a methodology of capturing operation and maintenance knowledge using spoken dialogue systems and integrates this knowledge within BIM models for future use by the building stakeholders. The following section will first introduce the spoken dialogue systems.

\section{Spoken Dialogue Systems (SDS)}

The motivation to develop SDS has been based on the need of having more effective user interfaces to computers to reduce the training and efforts required to enter/retrieve data and provide users with additional flexibility to perform tasks. SDS are human-computer interactive systems that use human natural language speech in input and output modalities to complete a task or solve a problem. Over the years, several specific dialogue systems have been developed in many fields such as: in weather reports (e.g. Zue et al. (2000)), tourism and navigation (e.g. Janarthanam et al (2013)), and in more complex domains like Self-Help where the system engages the user in technical tasks like troubleshooting by requesting information and providing instructions (e.g. William and Young (2007)). If developed with KM systems, SDSs can act as interactive expert agents that seek answers from the users for questions specific to the problem and help searching for solutions from the previously stored knowledge cases, which is the purpose of this research.

Generally, the use of voice-based technology, such as Interactive Voice Response (IVR) technology, has been tried in construction with limited applications. Abdel-Monem and 
Hegazy (2013) have integrated a cloud-based IVR service with a customised scheduling application to enhance progress tracking of projects. Abudayyeh (1997) has also attached voice-recorded files to various activities. Voice-based technology has also been used in voice commands to facilitate bridge inspection documentation (Sunkpho and Garrett, 2000). Tsai (2009) used voice recognition to record and update site-material logs. However, the application of voice-based techology with BIM and KM systems have not been tested in construction before.

A typical SDS includes: speech recognition, parsing and understanding, dialogue management, utterance generation and speech synthesis modules, as shown in Figure 1. The user's utterance (in the form of acoustic speech signals) is converted to a stream of words by the automatic speech recogniser module. The semantic parser is a Natural Language Understanding (NLU) module converts the stream of words into semantic frames called "Dialogue Actions" which give meaning representations of the user's utterances. The user dialogue actions are analysed by the Dialogue Manager (DM) and an appropriate response is formulated in consultation with the current state of the dialogue and the backend application. The system speech action produced by the DM is translated into utterance form by the Natural Language Generator (NLG) module and then is converted into acoustic signals by the speech synthesizer (or Text-To-Speech (TTS)) module.

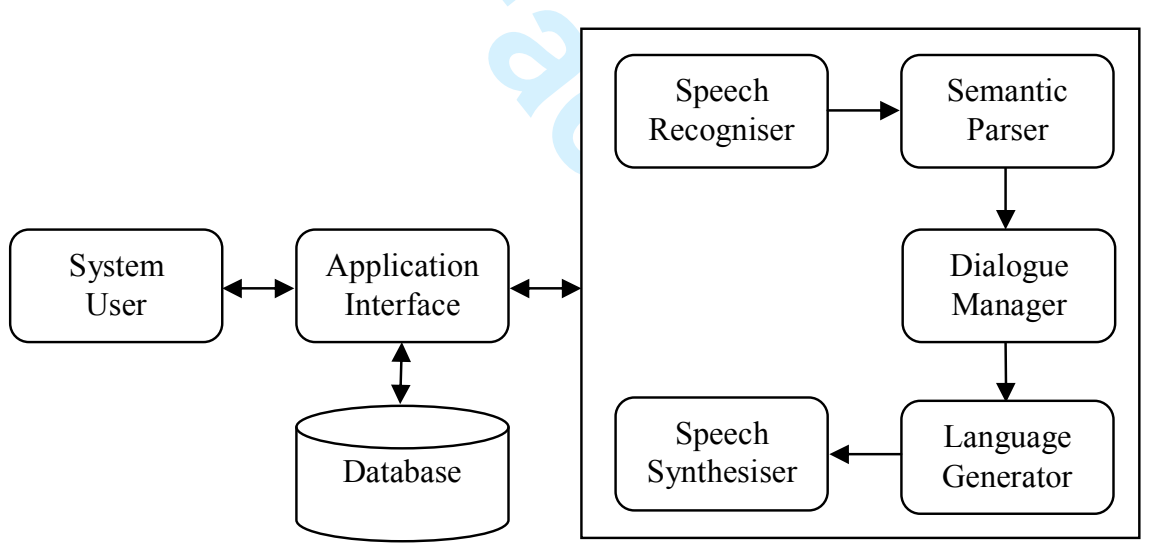

Figure 1. Spoken Dialogue System - Architecture

However, designing a dialogue system is more than putting together these modules. Although these modules are necessary, what makes a dialogue system natural and effective is its ability to coordinate these modules in a natural conversation with its dialogue partner. This task is the responsibility of the DM which manages the conversation using a plan, called the dialogue policy (or dialogue strategy) that maps any dialogue state to a dialogue action. The dialogue state maintains the system's knowledge, beliefs and observations of its environment (i.e. its current user), dialogue history, and goals. An enriched state may also contain the modality of interaction, and the user's profile containing his level of expertise, and cooperativeness. Speech actions that the DM can select include for example, greeting the user, requesting more information, presenting the results of the task, confirming existing information, and closing the dialogue. Dialogue policies can be manually coded for different tasks and situations. When there is a large number of factors affecting the dialogue (i.e. larger state space), manual coding can be difficult. One of the solutions to this problem is to learn the policies from human-human or human-machine dialogue data. For this research, SDS will 
be used to capture building maintenance knowledge and integrate this captured knowledge into the BIM model of the building. The following section will illustrate the main modules of the proposed system.

\section{System Modules}

The architecture of the proposed system, shown in Figure 2, integrates three modules: the BIM module to capture/retrieve the information of the building, the SDS module to verbally capture the maintenance-related knowledge, and the Case-Based Reasoning (CBR) knowledge module to analyse/retrieve the knowledge gained when dealing with buildings maintenance operations.

The BIM module is used to develop the virtual building model (Autodesk Revit is the BIM environment used for this research). Further parameters have been added to the BIM model for the building elements that comprises knowledge case details and categorisation which are mainly adopted from the taxonomy developed for this research, as shown in Table 1. Capturing knowledge/information cases involves filling the fields of these parameters with case details. These details are to be converted from the dialog captured by the SDS, which will be then processed by the CBR knowledge module. Further details on the taxonomy adopted for the knowledge cases in the proposed system can be found elsewhere; Motawa and Almarshad (2013).

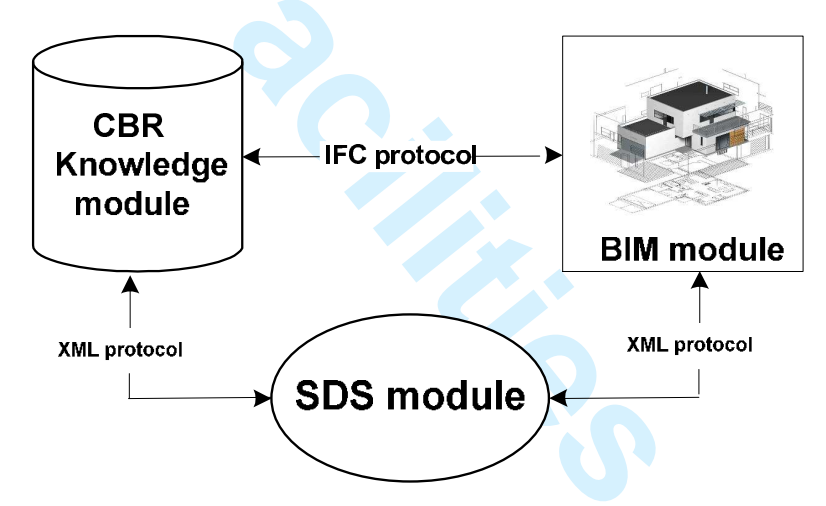

Figure 2. Main modules of the proposed system

In the developed prototype of the SDS module, there are dialogue manager and a speech synthesiser module. The prototype does not include semantic parsing and utterance generation modules for experimental purposes at this stage of the study. The cloud-based SDS service "Google Web Toolkit (2014)" has been integrated with the developed system. The cloud service has been programmed to capture the knowledge cases. The dialogue manager module uses a script in which the questions are prepared and coded in eXtensible Markup Language (XML). This script consists of a series of questions concerning the maintenance issue that will be put to the expert, the list of questions used are shown in Table 2. The expert can answer these questions using speech input. Each question is displayed on the screen as well as spoken to the expert using a speech synthesis module. The answers are converted into text strings by the speech recognition module. These recognised text strings are then stored in the knowledge base as a XML script linked to the appropriate component of the BIM model, an example of the XML script of answers is shown in Figure 3. The knowledge base expands by capturing various maintenance cases which enable the CBR module to acquire the closest case when searching the knowledge base for new problems. 
Table 1: Parameters of the knowledge case in the BIM module

\begin{tabular}{lll}
\hline Parameter name & Description \\
\hline 1. & Description & Problem description. \\
2. & Classification & The technical classification of the problem. \\
3. & Domain & The issue that the knowledge is related to. \\
4. & Location & The location where the problem occurred. \\
5. & Element & The element affected by the case. \\
6. & Root causes & Description of the root causes of the problem \\
7. & Reaction/Solution & The reaction/solution to the case. \\
8. & Side-effect & Description of any additional or follow-up operations \\
9. & BIM info & The BIM related information to the problem. \\
10. & Other info & Other related information to the problem \\
\hline
\end{tabular}

Table 2: List of questions for each maintenance case

1. Briefly describe the problem reported.

2. Who reported the problem to you?

3. Where did the problem occur? Which building, floor, zone and room?

4. What information from BIM did you find useful in your investigation?

5. What other information did you collect during investigation? Please also state the source of such information?

6. What are the components that are affected by this problem?

7. What was the root cause of the problem?

8. What solution did you propose to address the problem(s)?

9. What side-effects do you expect to happen following the implementation of the solution? What should be done if they happen?

10. Please add any other information is relevant to this case.

In order to distinguish between cases in the CBR module, certain attributes were assigned to the parameters of the taxonomy of the knowledge cases in order to query solutions of similar cases from the stored cases in the system. Dissimilarities between such attributes will lead to matching differences between the knowledge cases. Nearest-neighbour technique is implemented to retrieve the most similar case to a query by identifying and ranking the cases. In order to use CBR in problem solving, a weighting score should be assigned to each attribute of a knowledge case. Assigning these scores are based on the influence of an attribute on the retrieving process, further details on the methodology and the algorithm adopted for the CBR module can be found elsewhere; Motawa and Almarshad (2015).

The system uses an IFC protocol to integrate building models with the knowledge system. When uploading a BIM model for a particular building, the developed IFC protocol extracts the building details from the BIM environment including the classified knowledge for maintenance cases which are then organised and stored in the knowledge base to be later searched for solutions.

$<\mathrm{xml}>$

$<$ case component_id=""'> 
Figure 3. Example of the XML script for the maintenance cases

\section{CASE STUDY}

The proposed system has been applied to maintain a complex of commercial buildings, as shown in Figure 4. The validation stage has been conducted by testing the usage of the developed system by buildings professionals. When the expert (e.g. Facility managers) identifies the building element in the BIM model that has a problem and needs maintenance or repair, all details of the element and the added parameters will be populated. In addition, the required details of the maintenance case are acquired by the system using the dialogue interface, as shown in Figure 5. In contrast to existing approaches where experts are asked to type in their experiences in dealing with the issue into a text box, the system acquires expert knowledge as an interviewer and presents the expert with a series of questions concerning the different dimensions of the problem at hand. Instead of typing the answers to the questions which are at the same time the required input in the parameter fields, the expert can switch to the voice mode of the system and answer the questions one by one. The answers to the questions will be then converted by the SDS module and stored into the knowledge base. Once all questions are answered, the user can search the knowledge base to retrieve the closest stored case to the new one and investigate the old adopted solutions. This is done using the developed CBR module. An example of the search results for this particular problem is shown in Figure 6.

The proposed system has the ability to seek relationships between cases of several elements using the intelligent features of BIM objects. By tracing history of work and identifying related problems, this feature can provide professionals with a comprehensive understanding of issues related to their maintenance works. The proposed system identifies the spatial relationships between elements that are provided by the IFC schema. The system then clusters elements along with the associated cases into groups. Whenever a case has been searched and demonstrated, related cases of the same spatial group are presented. Figure 7 illustrates an example of the system output for this particular maintenance case and details of related cases.

During the validation stage, the interviewed professionals acknowledged the novelty of the developed system and its functions. The major issue raised by the interviewed professionals was about the need to improve the SDS technicality functions to capture and interpret the 
answered questions. This should be made by further developing the semantic parser. There are also some issues about the usage of the system within noisy construction sites. The raised issues from this feedback are actually among the main research areas within the SDS field for further development.

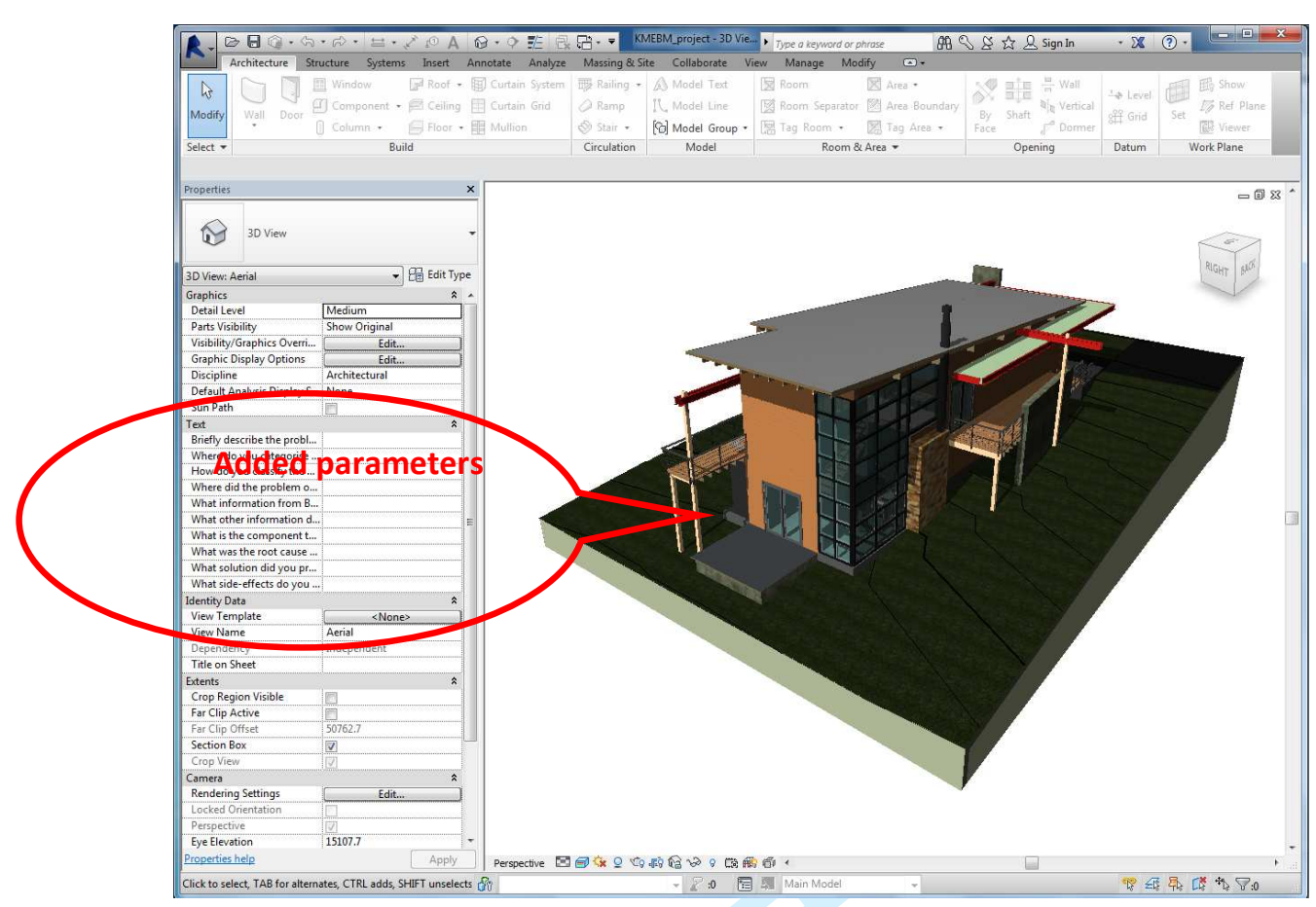

Figure 4. The BIM model of the case study building (output from Revit)

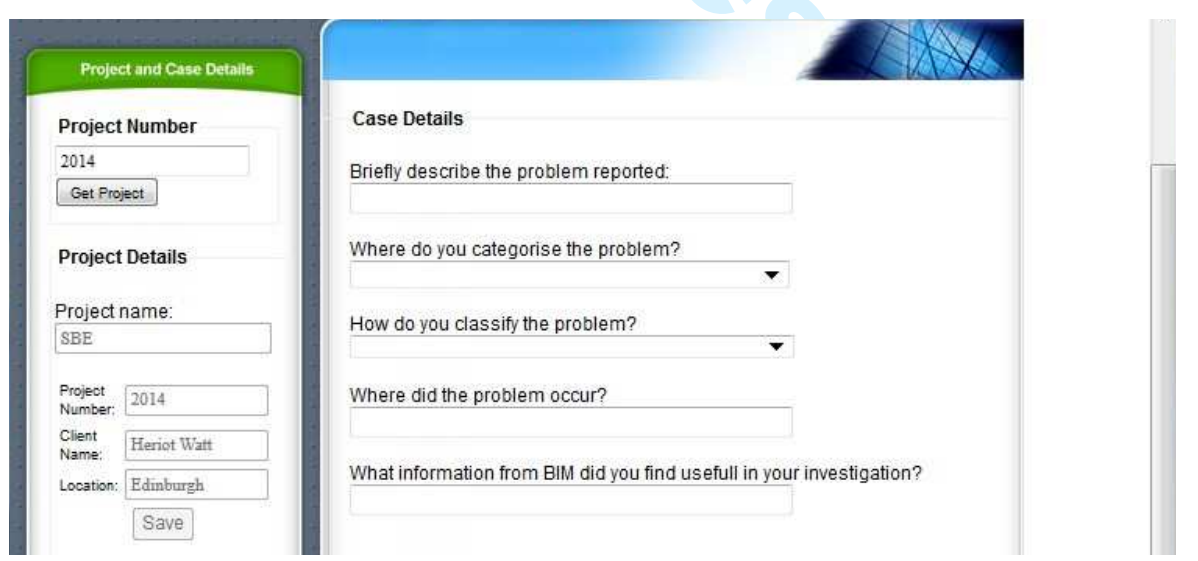

Figure 5. The dialogue interface to capture the knowledge case 


\section{Case Based Reasoning Results:}

01. Demolition. Dismantling and Remoral

02. Assembly and Installation

01. Earth works

02. Concrete Works

03. Masoner Works

04. Plaster Works

05. Steel Works

$\frac{05 \text {. Aluminium Works }}{06 \text {. }}$

07. False Ceiling Works

08. Roof Covering Works

09. Flooring and Cladding Works

04. Bathroom Hardware and

Sanitary Works

05. Water Supply Works

10. Insulation layers for Wetness.

Humidity and Heat

11. Wood Works

12. Paints Works

14. Construction. Design and

Landscaping Works

01. Labour and Technicians

02. Machinery and Equipment

01. HVAC Works

02. Mechanical Works

\section{Figure 6. The results of search the knowledge base using the CBR module}

Technical

+1. Demol. \& Assemb. +2. Civil works

+3. Mechanical works

+4. Electrical works

+6. External works
Knowledge Case Details:

Project Details

Project name: SBE

Client Name: Heriot Watt

Location:

Project Number: 2014

Case Details

problem:

Door problem

Cause of Problem:

testing root case of the problem

Solution:

Remove the inside doorknob by locating the button (like our model), metal tab or wire clip on the side of the knob that acts as a release. Using an awl

or small screwdriver, push the button in all the wayX97and at the same

timeX97pull off the handle. Remove the door rose by inserting a small

screwdriver into the small notch in the rose and twisting the tool to pop it

off. Be careful so you don

Category: 06. Aluminium Works

Class: BIM

Author Name: Mr. Almarshad

Attachments:

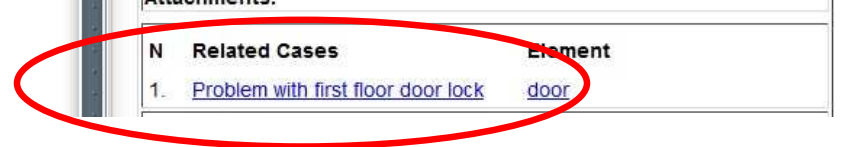

Figure 7. System output for a particular maintenance case and related cases 


\section{CONCLUSIONS}

Big data can be used to improve the development of the next generation of buildings and services. In this respect, BIM will be a major factor in this development. However, BIM largely considers structured data and allow the access to multiple databases. Therefore, new analytics methodology are sought to allow the analysis of unstructured data. For this research, an intelligent knowledge-based BIM system integrated with Spoken Dialogue System has been developed to capture and retrieve maintenance related information and knowledge of buildings. The advancements in Dialogue technology to handle data from various input and output modalities to generate a conversational interface was employed in the proposed system. The system acts as an interactive expert agent that seeks answers from the user for questions specific to the problem and help searching for solutions from the previously stored knowledge cases. The main conclusion from the study is that the practices of monitoring and maintaining buildings performance can be improved by integrating SDS with the knowledgebased BIM systems which enable easier capture and search for solutions to new problems with a more comprehensive retrieval of information. The integration of SDS for data capturing with case-based reasoning for data retrieval into BIM systems will also facilitate the complexity of using BIM systems which in practice needs sophisticated filtering techniques that might confuse the users of BIM systems. As buildings life may last for quite a number of decades and with the available variety of data collection tools such as: mobile devices, social media, smart meters, sensors, satellites, camera footage, traffic flow reports, etc., various data can be captured on performance and behaviour of operations, which can provide huge feedback for better buildings management. This can help design and operation teams to manage buildings, spaces, and services more efficiently. The data capture tools (including SDS) can now provide granular real-time data about utilization patterns which can improve the maintenance of buildings services and operations. The future work on this developed system is to integrate semantic parsing and utterance generation modules to the current version to convert the system interface into a full fledged dialogue system. The parsing capabilities will assist the CBR module in searching to acquire essential information using the speech modality. The utterance generation module will be used to generate customised responses from the database records based on information provided by users.

\section{REFERENCES}

Abdel-Monem, M. and Hegazy, T. (2013). Enhancing construction as-built documentation using interactive voice response. J. Consturction Engineering and Management, ASCE, 139(7), pp 895-898.

Abudayyeh, O. Y. (1997). A multimedia construction delay management system. J. Microcomput. Civil Eng., 12(3), pp 138-192.

Addor, M. and Santos, E. (2014). BIM Design Coordination Room Infrastructure: Assessment of Communication Activities. International Conference on Computing in Civil and Building Engineering, Orlando, Florida, United States, June 23-25, 2014: pp. 1-8.

Akhurst, P. \& Gillespie, N. (2006). "Sydney Opera House Facilities Management Exemplar Project." In: FMA Conference. 1 June 2006.

Ali, N., Sun, M., Petley, G. \& Barrett, P. (2002). "Improving the Business Process of Reactive Maintenance Projects." Facilities. 20 (7). p.p. 251-261. 
Ali, N., Sun, M., Petley, G.J., Barrett, P. \& Kagioglou, M. (2004). "MoPMIT: A Prototype System for Reactive Maintenance Projects in the UK." Journal Alam Bina. 6 (2)

Barrett, P. and Baldry, D. (2003). Facilities Management: Towards Best Practice, 2nd, Blackwell Science, Oxford, 2003.

Becerik-Gerber, B., Jazizadeh, F., Li, N. \& Calis, G. (2012). "Application Areas and Data Requirements for BIM-Enabled Facilities Management." Journal of Construction Engineering and Management. 138 (3). p.p. 431-442.

Caldas, C., Soibelman, L., and Gasser, L. (2005). "Methodology for the Integration of Project Documents in Model-Based Information Systems." J. Comput. Civ. Eng., 19(1), 25-33.

Chen, H., Chiang, R. H. L., and Storey, V. C., 2012, "Business Intelligence and Analytics: From Big Data to Big Impact," MIS Quarterly, 36(4), 1165-1188.

Chien-Ho, Ko. (2009). RFID-based building maintenance system, Automation in Construction, 18 (3), 275-284.

Courtney, M. (2012). "Puzzling out big data," Engineering \& Technology, 7(12), 56-60.

Fong, P.S.W. \& Wong, K. (2009). "Knowledge and Experience Sharing in Projects-Based Building Maintenance Community of Practice." International Journal of Knowledge Management Studies. 3 (3/4). p.p. 275 - 294.

Google Web Toolkit (2014). http://www.gwtproject.org/

Hao, Q., Xue, Y., Shen, W., Jones, B. \& Zhu, J. (2010). "A Decision Support System for Integrating Corrective Maintenance, Preventive Maintenance, and Condition-Based Maintenance." In: Construction Research Congress. 8 May 2010, Banff, Alberta.

Janarthanam, S. and Lemon, O. and Bartie, P. and Dalmas, T. and Dickinson, A. and Liu, X. and Mackaness, W.and Goetze, J. (2013). "Evaluating a City Exploration Dialogue System with Integrated Question-Answering and Pedestrian Navigation." Proc. of ACL 2013, Bulgaria.

Jiao, Y., Wang, Y., Zhang, S., Li, Y., Yang, B. \& Yuan, L. 2013. A cloud approach to unified lifecycle data management in architecture, engineering, construction and facilities management: Integrating BIMs and SNS. Advanced Engineering Informatics, 27, 173-188.

Lee, S. \& Akin, Ö. (2011). "Augmented Reality-Based Computational Fieldwork Support for Equipment Operations and Maintenance." Automation in Construction. 20 (4). p.p. 338352.

Legner, C. and Thiesse, F. (2006) RFID-based maintenance at Frankfurt airport, IEEE Pervasive Computing 5 (1), 34-39.

Lepkova, N. \& Bigelis, Z. (2007). "Model of Facilities Management Consulting Knowledge System." In: 9th International Conference on Modern Building Materials, Structures and Techniques, May 16-18. 16 May 2007, Vilnius, Lithuania.

Li, j., Wang, X., Wang, J., Guo, J., Zhang, S. \& Jiao, Y. 2014. A Project-based Quantification of BIM Benefits. International Journal Of Advanced Robotic Systems 11, 1-13.

Mao, W., Zhu, Y. \& Ahmad, I. 2007. Applying metedata models to unstructured content of construction documents: A view-based approach. Automation in Construction, 16, $242-$ 252.

McKinsey Global Institute "MGI" (2011). " Big data: The next frontier for innovation, competition, and productivity", June 2011, Copyright (C) McKinsey \& Company. Available at: http://www.mckinsey.com/insights/business technology/big data the next frontier_for i nnovation, accessed on 15/06/2015

McMalcolm, J. (2015). "Companies must not overlook big data security needs", available at http://www.businessreviewcanada.ca/technology/1225/Companies-must-not-overlook-bigdata-security-needs, accessed on 10/06/2015. 
Mello, R., Leite, L. R. and Martins, R. A. (2014), Is Big Data the Next Big Thing in Performance Measurement Systems?, In Y. Guan and H. Liao, eds. Proceedings of the 2014 Industrial and Systems Engineering Research Conference, Montréal, Canada, May 31 - June 3, 2014

Motawa, I and Almarshad, A (2015). "Case-based reasoning and BIM systems for asset management", Built Environment Project and Asset Management, Vol. 5(3), pp.233 - 247

Motawa, I and Almarshad, A (2013). "A knowledge-based BIM system for building maintenance." Journal of Automation in Construction, Volume 29, January 2013, Pages 173-182.

Motawa, I. and Carter, K. (2013). "Sustainable BIM-based Evaluation of Buildings." Procedia - Social and Behavioural Sciences Journal, Volume 74, pp. 116-125.

Oxford English Dictionary (2015). Available at: http://www.oxforddictionaries.com/definition/english/big-data

Parise, S., Iyer, B. and Vesset, D. (2012), Four strategies to capture and create value from big data, Issues: July/ August 2012, Ivey Business Journal, ISSN 1492-7071

Shrestha, J. (2013) "Big Data, Predictive Analytics, and Data Visualization in The Construction Engineering" CCEE Graduate Student Research Showcase and Poster Competition. Town Engineering Building. Dec. 2013. Available at: http://works.bepress.com/joseph_shrestha/2

Sunkpho, J. and Garrett, J. H. (2000). Opportunities to use speech recognition for bridge inspection. Proc. of Construction Congress VI, ASCE, Reston, VA.

Tien, J. M., 2013, "Big Data: Unleashing information," Journal of Systems Science and Systems Engineering, 22(2), 127-151.

Tsai, M. K. (2009). Improving communication barriers for on-site information flow: an exploratory study. J Adv. Eng. Inf., 23(3), 323-331.

Waller, M. A., and Fawcett, S. E., 2013, "Data Science, Predictive Analytics, and Big Data: A Revolution That Will Transform Supply Chain Design and Management," Journal of Business Logistics, 34(2), 77-84.

Williams, J. and Young, S. (2007). "Partially Observable Markov Decision Processes for Spoken Dialog Systems.” Journal of Computer Speech and Language, volume 21(20), pages 231-422.

Zhu, Y., Mao, W. \& Ahmad, I. 2007. Capturing implicit structures in unstructured content of construction documents. Journal of Coumputing in Civil Engineering, 21, 220-227.

Zue, V. and Seneff, S. and Glass, J. and Polifroni, J. and Pao, C. and Hazen, T. and Hetherington, L. (2000). "JUPITER: A Telephone-Based Conversational Interface for Weather Information.” IEEE Transactions on Speech and Audio Processing, volume 8(1). 